> Les anomalies des protéines de l'enveloppe nucléaire sont responsables d'affections musculaires, cardiaques, nerveuses, du tissu adipeux et de vieillissement prématuré (Cahiers de Myologie, 2010 ; 3 : 24-33). Depuis 2000, le réseau français «des dystrophies musculaires d'Emery-Dreifuss et autres pathologies de l'enveloppe nucléaire » organise une réunion annuelle. La $14^{\mathrm{e}}$ édition a été organisée conjointement avec le « réseau italien des laminopathies $\gg$. Cette $1^{\text {re }}$ réunion franco-italienne consacrée à ces affections a été l'occasion d'interactions fructueuses entre divers acteurs de la recherche fondamentale et clinique travaillant sur ces maladies génétiques rares. <
La première réunion franco-italienne des laminopathies et autres pathologies liées à l'enveloppe nucléaire s'est tenue à l'Hôpital de La Timone à Marseille, les 15 et 16 janvier 2015. Cette réunion organisée par Annachiara De Sandre Giovannoli et Nicolas Lévy et les responsables des réseaux italiens et français, respectivement Giovanna Lattanzi (Bologne) et Gisèle Bonne, France Leturcq et R. Ben Yaou (Paris), s'est déroulée sur deux jours en présence de 108 participants français et italiens, mais également portugais et espagnols. La première journée destinée aux scientifiques a porté sur les développements récents des recherches précliniques et cliniques, alors que la deuxième journée ouverte également aux patients et à leurs familles a abordé les approches thérapeutiques en cours de développement ainsi que les registres et les bases de données.

\section{Laminopathies : recherche préclinique}

Après la présentation des objectifs et organisations respectifs des réseaux italiens et français, la session portant sur les recherches précliniques a débuté par un tour d'horizon des mécanismes impliqués dans le vieillissement normal et pathologique (Carlos Lopez-Otin, chercheur à I'Université d'Oviedo, Espagne), suivi d'un exposé sur divers modèles

Vignette (images de noyaux normaux de fibroblastes humains et anormaux en présence de mutation des lamines $A / C$ - (c) Gisèle Bonne).

\title{
Première réunion franco-italienne des laminopathies et autres pathologies liées à l'enveloppe nucléaire
}

Rabah Ben Yaou ${ }^{1,4}$, Annachiara De Sandre-Giovannoli², France Leturcq ${ }^{1,3}$, Nicolas Lévy ${ }^{2}$, Gisèle Bonne ${ }^{1}$

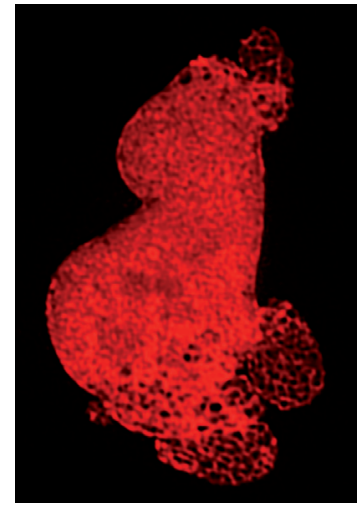

cellulaires dérivés de cellules de patients atteints de laminopathies. Parmi eux, des cellules souches pluripotentes induites (iPS cells) dérivées de patients présentant une progéria de Hutchison-Gilford ont été utilisées pour la mise en place de preuves de principes: I'un pour un modèle de criblage de molécules pharmacologiques à haut débit présenté par Xavier Nissan (I-Stem, Évry) ; l'autre pour l'étude de la différenciation vers le lignage musculaire lisse vasculaire présenté par Lino Ferreira (Université de Coimbra, Portugal). L'utilisation de précurseurs myogéniques issus de biopsies musculaires cultivées en 3D a été présentée par Catherine Coirault (Centre de recherche en Myologie, Paris) comme modèle permettant d'étudier des défauts de mécano-sensibilité. Des travaux basés sur l'exploration in vivo des différents modèles animaux ont également été présentés, avec un focus sur l'atteinte cardiaque et l'atteinte hypothalamique observées respectivement chez deux modèles murins de laminopathies et présentés par Antoine Muchir (Centre de Recherche en Myologie, Paris) et par Claudia Cavadas (Université de Coimbra, Portugal).

De nouvelles approches thérapeutiques des laminopathies ont été ensuite abordées, avec entre autres : l'inhibition de la production de progérine dans les vieillissements prématurés par Camilla Pellegrini (Bologne, Italie) : la clairance augmentée de la progérine par Karim Harhouri (Inserm UMRS 910, Marseille); le trans-épissage dans les laminopathies du muscle strié par 


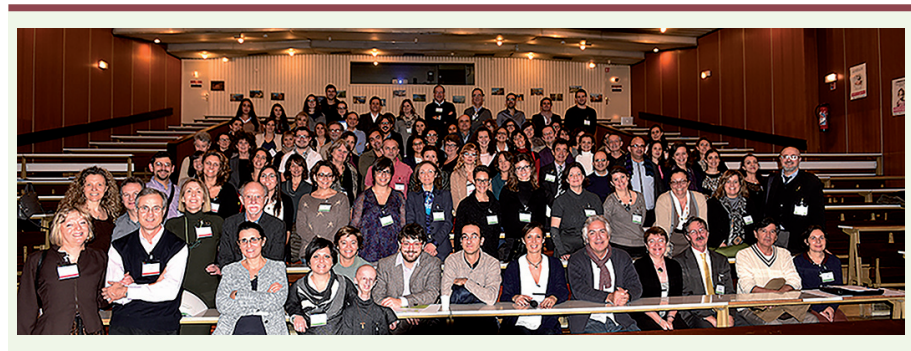

Figure 1.

Feriel Azibani (Centre de recherche en Myologie, Paris) et des traitements à base de Metreleptin ou d'inhibiteurs de la protéase dans les lipodystrophies partielles, formes de laminopathies touchant le tissu graisseux, par Camille Vatier et Corinne Vigouroux (Inserm UMRS 938, Hôpital Saint-Antoine, Paris). L'exploration de nouveaux biomarqueurs a fait l'objet d'une session spécifique avec la caractérisation des altérations de la dynamique chromatinienne et des profils de cytokines par l'équipe de Giovanna Lattanzi (Bologne, Italie) et la dérégulation de micro-ARN par Patrice Roll (Inserm UMRS 910, Marseille).

\section{Dystrophie musculaire d'Emery-Dreifuss : recherches précliniques}

La première journée a également été l'occasion de revenir sur les formes liées à l'X de la dystrophie musculaire d'Emery-Dreifuss, dues à des mutations des gènes codant l'émerine et la protéine FHLl. Isolin Herraa, doctorante de l'équipe de biologie structurale de Sophie Zinn-Justin (CEA, Saclay) a présenté l'impact de l'oligomérisation de l'émerine sur les interactions entre lamines et actine. Esma Ziat, doctorante dans l'équipe de Gisèle Bonne (Institut de myologie, Paris) a présenté l'étude des différentes isoformes de FHLl au cours de la différenciation myogénique dans les contextes normal et pathologique.

\section{Recherche clinique des pathologies de l'enveloppe nucléaire}

Plusieurs sujets de recherche clinique concernant ces affections ont été abordés. Une mise à jour des aspects cliniques observés dans les émerinopathies a été présentée par France Leturcq (Hôpital Cochin, Paris) et Rabah Ben Yaou (Institut de myologie, Paris). Lorenzo Maggi (Milan, Italie) a rapporté l'expérience du réseau italien sur une grande cohorte de patients atteints de laminopathies musculaires et cardiaques. Nicola Carboni (Nuoro, Italie) a, quant à lui, insisté sur l'imagerie musculaire et son intérêt dans le diagnostic précoce de la dystrophie musculaire d'Emery-Dreifuss. Saida Ortolano (Vigo, Espagne) a détaillé les aspects cliniques observés dans une famille atteinte d'une forme de myopathie des ceintures liée à des mutations de la transportine 3, protéine de l'enveloppe nucléaire récemment identifiée. Enfin, MarieChristine Vantyghem (CHU de Lille) a présenté une étude sur l'évaluation de l'irisine chez des patients souffrant de lipodystrophie partielle.

Le deuxième jour en présence des patients et leurs familles, après une présentation de la grande diversité clinique et génétique de ces affections par Raoul Hennekam (Université d'Amsterdam, Pays-Bas), les divers volets de la prise en charge de ces maladies ont été abordés. Les formes congénitales de dystrophie musculaire liées aux lamines $A / C$ sont particulières par leur sévérité. Susana Quijano-Roy (Hôpital Raymond Poincaré, Garches) en a détaillé la présentation clinique et les traitements actuellement disponibles. Guiseppe Boriani (Milan,
Italie) a rappelé les principes de la prise en charge cardiologique de ces affections à la lumière des dernières connaissances moléculaires et l'utilité de la mise en place précoce de dispositifs intracardiaques de type pacemaker et défibrillateur automatique implantable. Corinne Vigouroux a montré les résultats préliminaires d'un essai thérapeutique par la leptine dans une cohorte de patients atteints de lipodystrophie liée aux lamines A/C. Annachiara De Sandre-Giovannoli (Inserm UMRS 910, Marseille) a, quant à elle, décrit les travaux de préparation du futur essai clinique utilisant une approche antisens dans les syndromes de vieillissement prématuré liés à l'accumulation de progérine. Enfin, Luigi Ravagnan a exposé la politique actuelle de la Fondation Maladies Rares pour le soutien de la mise en place d'essais thérapeutiques dans les maladies rares.

\section{Registres et bases de données}

Après une table ronde qui a laissé libre cours aux échanges entre médecins, chercheurs, patients et association de patients, la réunion a été clôturée par une session dédiée aux registres et bases de données. Gaëlle Blandin de l'équipe de Christophe Béroud (Inserm UMRS 910, Marseille) a rappelé l'utilité des registres de patients comme outil rassemblant en un lieu unique des données cliniques, moléculaires et épidémiologiques de patients. Sara Benedetti (Milan, Italie) a présenté les aspects cliniques des cardio-laminopathies avec comme perspective la mise en place d'un registre dédié. David Araujo-Vilar (Santiago de Compostela, Espagne) a détaillé le fonctionnement du consortium européen ECLip dédié aux lipodystrophies. Enfin, Gisèle Bonne a décrit l'observatoire français des patients atteints de laminopathies et émerinopathies (OPALE) et a évoqué la possibilité de mettre en place un registre franco-italien.

\section{Conclusion}

En conclusion, ces deux journées très riches en échanges et discussions, il est envisagé d'organiser non seulement une deuxième réunion conjointe des réseaux français et italiens, mais aussi d'ouvrir ces réunions plus largement à nos autres collègues européens, notamment ceux de la péninsule ibérique déjà bien représentés lors de cette première édition. Nul doute que cette première édition sera la première d'une longue série, tant le champ des laminopathies et des maladies de l'enveloppe nucléaire est riche, varié et en perpétuelle évolution. $\diamond$ First Italo-French meeting on laminopathies and other pathologies related to the nuclear envelope

\section{LIENS D'INTÉRÊT}

Les auteurs déclarent n'avoir aucun lien d'intérêt concernant les données publiées dans cet article.

\section{TIRÉS À PART}

G. Bonne 